\title{
Cross Interaction Between Ilyonectria mors-panacis Isolates Infecting Korean Ginseng and Ginseng Saponins in Correlation with Their Pathogenicity
}

\author{
Mohamed El-Agamy Farh, Yeon-Ju Kim, Priyanka Singh, and Deok-Chun Yang
}

First, second, and fourth authors: Graduate School of Biotechnology and Ginseng Bank, College of Life Science, Kyung Hee University, Yongin, 446-701, Republic of Korea; and third and fourth authors: Department of Oriental Medicinal Biotechnology, College of Life Sciences, Kyung Hee University, Republic of Korea.

Accepted for publication 28 November 2016.

\begin{abstract}
Ilyonectria mors-panacis belongs to I. radicicola species complex and causes root rot and replant failure of ginseng in Asia and North America. The aims of this work were to identify I. mors-panacis that infect Korean ginseng using molecular approaches and to investigate whether their aggressiveness depends on their ability to metabolize ginseng saponins (ginsenosides) by their $\beta$-glucosidases, in comparison with other identified Ilyonectria species. Fourteen isolates were collected from culture collections or directly isolated from infected roots and mainly identified based on histone H3 (HIS H3) sequence. Among them, six isolates were identified as I. mors-panacis while others were identified as I. robusta and I. leucospermi. The pathogenicity tests confirmed that the isolates of I. mors-panacis were significantly more aggressive than I. robusta and I. leucospermi. The major ginsenosides in I. mors-panacisinfected roots were significantly reduced while significantly increased in

those infected with other species. In vitro, the isolates were tested for their sensitivity and ability to metabolize the total major ginsenosides (Total $\mathrm{MaG}$ ), protopanaxadiol-type major ginsenosides (PPD-type MaG), and protopanaxatriol-type major ginsenosides (PPT-type MaG). Unexpectedly, the growth rate and metabolic ability of I. mors-panacis isolates were significantly low on the three different ginsenoside fractions while those of $I$. robusta and I. leucospermi were significantly reduced on PPT-type MaG and Total MaG fractions and not affected on PPD-type $\mathrm{MaG}$ fraction. Our results indicate that major ginsenosides, especially PPT-type, have an antifungal effect and may intervene in ginseng defense during Ilyonectria species invasion, in particular the weak species. Also, the pathogenicity of $I$. mors-panacis may rely on its ability to reduce saponin content; however, whether this reduction is caused by detoxification or another method remains unclear.
\end{abstract}

Korean ginseng (Panax ginseng) is a member of the family Araliaceae and a well-known medicinal plant in Asian countries. The roots are commonly used in traditional Asian medicine to modulate blood pressure, improve metabolism, and strengthen the immune system. These effects have been shown to be elicited by ginsenosides, which are dammarane-type triterpenoid saponins with one or more sugar residues. Based on their sapogenins, which comprise the skeleton of the dammarane, ginsenosides are divided into two types: protopanaxadiol (PPD)- and protopanaxatriol (PPT)-type ginsenosides (Fig. 1). PPD-type ginsenosides have saccharide moieties at C-3 and C-20 [e.g., Rb1 1, Rb2 2, Rc 3, Rd 4, F2 5, compound Y (c-Y) 6, compound MC (c-MC) 7, compound-K (c-K) 8, and protopanaxadiol (PPD) 9] while PPTtype ginsenosides have saccharide moieties at C-6 and C-20 (e.g., Rg1 11, Re 10, Rf 12, $\operatorname{Rg} 2$ 13, and Rh1 14) (Kim et al. 2013). Ginsenosides are of interest in the medical field due to their multipharmaceutical effects (Leung and Wong 2010; Siraj et al. 2015), but their role in the environment has yet to be thoroughly investigated. In general, saponins exhibit antifungal activities against soil-borne fungi that inhabit the environment of saponin-producing plants (Crombie et al. 1986; Fewell and Roddick 1993; Morrissey and Osbourn 1999; Nicol et al. 2002; Papadopoulou et al. 1999). They exhibit antifungal action by binding to fungal steroids, ergosteroids (Armah et al. 1999; Keukens et al. 1995), possibly at a free 3 $\beta$-hydroxyl group (Steel and Drysdale 1988). Fungi that feed off saponin-producing

Corresponding authors: D.-C. Yang; E-mail address: dcyang@khu.co.kr, and Y.-J. Kim; E-mail address: yeonjukim@khu.ac.kr

*The $\boldsymbol{e}$-Xtra logo stands for "electronic extra" and indicates that three supplementary figures and four supplementary tables are published online.

(c) 2017 The American Phytopathological Society plants have been found to be less sensitive to the antifungal effects of saponins than nonpathogenic fungi. These saponin-resistant fungi produce saponin hydrolyzing enzymes to decrease the fungitoxicity of saponins (Morrissey and Osbourn 1999; Osbourn 1996). For example, Gaeumannomyces graminis var. avenae can detoxify avenacin-1, the main saponin of the oat plant, to a less toxic compound, and Septoria lycopersici (Osbourn et al. 1995), Verticillium albo-atrum (Pegg and Woodward 1986), Botrytis cinerea, Fusarium oxysporum f. sp. lycopersici (Ford et al. 1977), and Fusarium solani (Lairini and Ruiz-Rubio 1998) can detoxify $\alpha$-tomatine, the saponin of tomato plant, to innocuous compounds. Detoxification of saponins by pathogenic fungi has been found to be crucially important for the infection progress; mutant strains of those pathogens (which cannot hydrolyze saponins) are not able to infect their host plants. The relationship between saponins and the aggressiveness of fungal pathogens has sparked interest in the interactions between ginsenosides and ginseng pathogens. In a study by Nicol et al. (2003), ginsenosides stimulated the growth of Pythium irregulare, an oomycetous pathogen that causes damping-off of American ginseng seedlings (Panax quinquefolius), while inhibiting other nonpathogenic fungi. Growth stimulation by ginsenosides was attributed to the lack of natural sterols in the pathogen membrane (Nicol et al. 2003). The presence of the minor ginsenoside F2 in the cell membrane of $P$. irregulare after selective metabolism of PPD-type major ginsenosides (PPD-type MaG) (Yousef and Bernards 2006) and the positive proportional relationship observed between the pathogenicity of different isolates of $P$. irregulare taken from different plant sources and their ability to metabolize PPD-type MaGs to F2 (Ivanov and Bernards 2012) supported the hypothesis.

Cylindrocarpon destructans is a soilborne pathogenic fungus belonging to the family Nectriaceae (Hypocreales) (Rossman et al. 1999). It causes severe root rot in many hosts including ginseng plants (Booth 1966; Seifert et al. 2003; Sutherland et al. 1989; 
Unestam et al. 1989). C. destructans has also been reported to be associated with a mild disease in ginseng, namely rust disease (Hildebrand 1935). As with a number of other pathogenic fungi, C. destructans can be divided into weak or aggressive isolates on ginseng and other hosts (Hildebrand 1935; Lyr and Kluge 1968; Seifert et al. 2003). Aggressive isolates are usually associated with severe root rot disease, while weakly aggressive isolates are associated with rust disease (Hildebrand 1935; Seifert et al. 2003). Aggressive isolates were found to be highly adapted for ginseng infection with limited pathogenicity in other hosts, and were therefore named C. destructans f. sp. panacis (Seifert et al. 2003). The genus Cylindrocarpon was recently reclassified into four different genera: Ilyonectria, Neonectria/Cylindrocarpon s. str., Rugonectria, and Thelonectria. C. destructans was reclassified as I. radicicola (Chaverri et al. 2011). However, multigene molecular analysis, especially histone H3 (HIS H3), revealed a polyphyletic relationship among I. radicicola (Cabral et al. 2011; Lombard et al. 2013) isolates and hence, each group of isolates is considered to be a different species. The isolates reported as aggressive, namely C. destructans f. sp. panacis (Seifert et al. 2003), were genetically distinct from the other isolates and clustered in a distinct group named as I. mors-panacis, while isolates reported to be weakly aggressive (Seifert et al. 2003), clustered into three different species, I. panacis, I. robusta, and I. crassa (Cabral et al. 2011). Most of the identified isolates were isolated from American ginseng, and the Korean ginseng-infecting isolates have not yet been thoroughly characterized (Cabral et al. 2011). Many differences have been observed between the highly and weakly aggressive members of I. radicicola species complex, one of them being the enzymes they

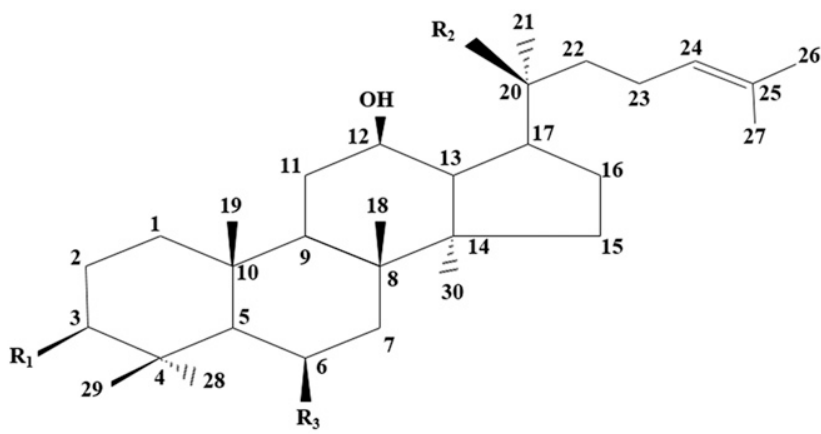

\begin{tabular}{|c|c|c|c|c|}
\hline Compound & Ginsenosides & $\mathrm{R}_{1}(\mathrm{C}-3)$ & $\mathrm{R}_{2}(\mathrm{C}-20)$ & $\mathrm{R}_{3}(\mathrm{C}-6)$ \\
\hline 1 & Rbl & $\mathrm{O}-\mathrm{glc}(2 \rightarrow 1) \mathrm{glc}$ & $\mathrm{O}-\mathrm{glc}(6 \rightarrow 1) \mathrm{glc}$ & $\mathrm{H}$ \\
\hline 2 & $\mathrm{Rb} 2$ & $\mathrm{O}-\mathrm{glc}(2 \rightarrow 1) \mathrm{glc}$ & O-glc $(6 \rightarrow 1)$ arap & $\mathrm{H}$ \\
\hline 3 & $\mathrm{Rc}$ & O-glc $(2 \rightarrow 1) \mathrm{glc}$ & O-glc $(6 \rightarrow 1)$ araf & $\mathrm{H}$ \\
\hline 4 & $\mathrm{Rd}$ & O-glc $(2 \rightarrow 1)$-glc & O-glc & $\mathrm{H}$ \\
\hline 5 & $\mathrm{~F} 2$ & O-glc & O-glc & $\mathrm{H}$ \\
\hline 6 & $c-Y$ & $\mathrm{OH}$ & O-glc $(6 \rightarrow 1)$ arap & $\mathrm{H}$ \\
\hline 7 & $\mathrm{c}-\mathrm{MC}$ & $\mathrm{OH}$ & O-glc $(6 \rightarrow 1)$ araf & $\mathrm{H}$ \\
\hline 8 & $\mathrm{c}-\mathrm{K}$ & $\mathrm{OH}$ & O-glc & $\mathrm{H}$ \\
\hline 9 & PPD & $\mathrm{OH}$ & $\mathrm{OH}$ & $\mathrm{H}$ \\
\hline 10 & $\mathrm{Re}$ & $\mathrm{OH}$ & O-glc & $\begin{array}{c}\text { O-glc }(2 \rightarrow 1) R \\
\text { ha }\end{array}$ \\
\hline 11 & Rg1 & $\mathrm{OH}$ & O-glc & O-glc \\
\hline 12 & $\mathrm{Rf}$ & $\mathrm{OH}$ & $\mathrm{OH}$ & $\begin{array}{c}\text { O- } \\
\operatorname{glc}(2 \rightarrow 1) \mathrm{glc}\end{array}$ \\
\hline 13 & Rg2 & $\mathrm{OH}$ & $\mathrm{OH}$ & $\begin{array}{c}\text { O-glc }(2 \rightarrow 1) R \\
\text { ha }\end{array}$ \\
\hline 14 & Rh1 & $\mathrm{OH}$ & $\mathrm{OH}$ & O-glc \\
\hline
\end{tabular}

Fig. 1. The chemical structures of the different PPD-type and PPT-type ginsenosides. Abbreviations: PPD-type, protopanaxadiol-type; PPT-type, protopanaxatrioltype; c-Y, compound Y; c-MC, compound MC; c-K, compound K; glc, $\beta$-Dglucopyranosyl; arap, $\alpha$-L-arabinopyranosyl; araf, $\alpha$-L-arabinofuranosyl; and Rha, $\alpha$-L-rhamnopyranosyl. produce. For example, the hydrolytic enzyme, pectinase and the oxidative enzyme, polyphenol oxidase were found to be produced by the highly aggressive members in higher quantities than weakly aggressive (Rahman and Punja 2005). Degradation of PPD-type MaG by I. radicicola species complex was reported (Zhao et al. 2012), but there is no evidence that the isolate used in this study is I. mors-panacis or that this activity correlates with the fungus pathogenicity. Therefore, the goal of the present study is to identify the isolates of the highly aggressive species, I. morspanacis infecting Korean ginseng using molecular approaches and then check whether their aggressiveness depends on the degradation of ginseng saponins compared with other identified, weakly aggressive Ilyonectria species.

\section{MATERIALS AND METHODS}

Microbial and plant materials. Healthy, 2-year-old ginseng roots for pathogenicity test were obtained from a private farm and kept at $4^{\circ} \mathrm{C}$ until artificial infection.

Eight isolates of the $I$. radicicola species complex isolated from Korean ginseng roots were obtained either from the Korean Agriculture Culture Collection (KACC), South Korea, or the NITE Biological Resource Center (NBRC), Japan, while an additional six isolates were isolated directly from infected 4-year-old cultivated ginseng collected from Yongin Province $\left(37^{\circ} 14^{\prime} 43^{\prime \prime} \mathrm{N} ; 127^{\circ} 04^{\prime} 56^{\prime \prime} \mathrm{E}\right)$, Gochang Province $\left(35^{\circ} 23^{\prime} 56^{\prime \prime} \mathrm{N} ; 126^{\circ} 33^{\prime} 26^{\prime \prime} \mathrm{E}\right)$, and Yeoncheon $\left(38^{\circ} 05^{\prime} 48^{\prime \prime} \mathrm{N}\right.$; $\left.127^{\circ} 04^{\prime} 30^{\prime \prime} \mathrm{E}\right)$, South Korea. Isolation was performed as described by Rahman and Punja (2005). A saprophytic fungus, Trichoderma spp. (ITS accession number KU238148) isolate was isolated from ginseng rhizosphere and used as a control for ginsenoside sensitivity and pathogenicity assays. All isolates were stored long-term in stock vials of synthetic nutrient broth (SNB) (Nirenberg 1976) supplemented with $10 \%$ glycerol at $-70^{\circ} \mathrm{C}$. All information about the I. radicicola species complex isolates used in this study is summarized in Table 1.

Saponin extract prepared from ginseng roots and ginsenoside standards Rb1 1, Rb2 2, Rc 3, Rd 4, c-K 8, Rg111, Re 10, Rg2 13, and Rh1 14 were obtained from the Ginseng Bank (Kyung-Hee University, Yongin, Korea). c-Y 6 and c-MC 7 standards were kindly provided by Professor Wan Taek Im (Hankyong National University, South Korea).

Molecular characterization of $I$. radicicola species complex isolates. The isolates were grown on potato dextrose agar (PDA; $\mathrm{MB}$ Cell) in the dark at $25^{\circ} \mathrm{C}$ for 10 days. Then, each isolate was cultured in $150 \mathrm{ml}$ of potato dextrose broth in the dark at $25^{\circ} \mathrm{C}$ with shaking $(150 \mathrm{rpm})$ for 10 days. The mycelia were collected by filtration, washed three times with water, freeze-dried, and kept at $-70^{\circ} \mathrm{C}$ as stock mycelia for DNA isolation. Twenty milligrams of freeze-dried mycelium of each isolate was crushed by plastic pestle and DNA was extracted using the DNeasy Plant Mini Kit (Qiagen) as instructed by the manufacturer. PCR amplification and sequencing of the HIS H3 were performed as follows: $1 \mu \mathrm{l}$ of genomic DNA was mixed with $7.5 \mu$ l of green mix (Genotech, South Korea), 5 pmol of each primer, and $5.5 \mu \mathrm{l}$ of water for a final volume of $15 \mu \mathrm{l}$. PCR reaction was carried out using MyCycler thermal cycler (Bio-Rad) under the following conditions: $95^{\circ} \mathrm{C}$ for $5 \mathrm{~min}$, followed by 40 cycles at $94^{\circ} \mathrm{C}$ for $30 \mathrm{~s}, 64^{\circ} \mathrm{C}$ for $30 \mathrm{~s}$, and $72^{\circ} \mathrm{C}$ for $80 \mathrm{~s}$, and a final elongation at $72^{\circ} \mathrm{C}$ for $10 \mathrm{~min}$. Forward and reverse primers were CYLH3F (5'-AGG TCC ACT GGT GGC AAG-3') and CYLH3R (5'-AGC TGG ATG TCC TTG GAC TG-3') (Crous et al. 2004). The purity of the desired bands was confirmed by electrophoresis. PCR products were purified using a commercial gel purification kit (Genotech, South Korea) and sequenced using the same primer pairs used for amplification by Genotech (Daejeon, Republic of Korea). Sequences were corrected using the SeqMan module of the Lasergene software package (DNAStar, Madison), and blasted on the GenBank database (http://blast.ncbi.nlm.nih. gov/Blast.cgi) for identification. 
Pathogenicity test. Pathogenicity test was performed as described by Kernaghan et al. (2007) with some modifications; each identified isolate (14 isolates of I. radicicola species complex and, for comparison, 1 isolate of Trichoderma spp.) were grown on PDA in the dark at $25^{\circ} \mathrm{C}$ for 10 days and then transcultured in clarified V8 broth in the dark at $25^{\circ} \mathrm{C}$ with shaking $(150 \mathrm{rpm})$ for 2 weeks. Each mycelial mat was then filtered, blended with $45 \mathrm{ml}$ of sterilized water, and thoroughly mixed with sterilized artificial soil to a final concentration of $40 \mathrm{ml} / \mathrm{kg}$. Soil mixed with sterilized water only at the same concentration was prepared as the untreated control. Infected soil samples were transferred to $15-\mathrm{cm}$ pots and then 2-year-old cultivated ginseng roots with similar lengths, widths, and weights (six roots/pot) were cultivated. Two pots were set up for each isolate as well as the control, and the experiment was conducted three times. Pots were incubated in a greenhouse $(22 \pm$ $2{ }^{\circ} \mathrm{C}, 12$-h photoperiod) for 4 weeks. To determine the disease severity index (DSI), infected roots were rated using the scale described in Supplementary Table S1 and Supplementary Fig. S1. DSI was normalized for each isolate using the following equation: DSI $=\left[\left(X_{1} \times 1\right)+\left(X_{2} \times 2\right)+\left(X_{3} \times 3\right)+\left(X_{4} \times 4\right)+\left(X_{5} \times 5\right)+\left(X_{6} \times 6\right)\right] /$ $\left(X_{1}+X_{2}+X_{3}+X_{4}+X_{5}+X_{6}\right)$, where $X_{1}, X_{2}, X_{3}, X_{4}, X_{5}$, and $X_{6}$ are the numbers of plants with rotting severity scales of $1,2,3,4,5$, and 6 , respectively.

Extraction of ginsenosides from infected roots. After assessing the pathogenicity of the isolates, roots were freeze-dried and used for investigating the ginsenosides content and any changes in levels due to infection. Ginsenosides were extracted as described by Kim et al. (2013) with some modifications; for each treatment, six roots inoculated with each isolate were ground into a powder using a mortar. The powder was placed in a round flask, mixed with $40 \mathrm{ml}$ of $80 \%$ methanol, and heated at $80^{\circ} \mathrm{C}$ using a Soxhlet water bath. After $2 \mathrm{~h}$, the methanolic extract was collected and evaporated at $45^{\circ} \mathrm{C}$ using a rotary evaporator. Dried samples were redissolved in $20 \mathrm{ml}$ of distilled water. The ginsenosides were extracted using $20 \mathrm{ml}$ of water-saturated $n$-butanol. The butanol layers were collected and evaporated at $55^{\circ} \mathrm{C}$ using a rotary evaporator and finally, the dried samples (crude ginsenosides) were redissolved in $1 \mathrm{ml}$ of methanol, filtered through $0.2-\mu \mathrm{m}$ filters, and subjected to high-performance liquid chromatography (HPLC) for ginsenoside quantification.

Preparation of ginsenoside fractions. Saponin extract from white ginseng root was used to prepare three fractions for in vitro antifungal and metabolism assays of ginsenosides: total major ginsenosides (Total MaG), PPD-type major ginsenosides (PPDtype $\mathrm{MaG}$ ), and PPT-type major ginsenosides (PPT-type MaG). The extract was first washed with water to remove sugars using open column chromatography with resin HP20 as the stationary phase. Then, Total MaG was obtained by eluting with the methanol: water (95:5, vol/vol). The PPD-type MaG fraction was separated from the PPT-type MaG fraction using the methanol gradient method described by Shehzad et al. (2011) with some modifications; total PPT-type MaG were eluted using methanol: water (65:35, vol/vol). Then, PPD-type MaG was eluted using methanol: water $(90: 10, \mathrm{vol} / \mathrm{vol})$. All fractions were collected and vacuum-dried, and ginsenosides were quantified in three replicates using HPLC.

Assessment of the antifungal activity of ginsenosides. The susceptibility of isolates of the $I$. radicicola species complex and Trichoderma spp. to the three fractions of ginsenosides (Total$\mathrm{MaG}$, PPD-type $\mathrm{MaG}$, and PPT-type $\mathrm{MaG}$ ) was evaluated by estimating the colony growth diameter in V8 agar supplemented with each fraction compared with the untreated control. Each fraction was supplied in V8 agar at $4 \mathrm{mg} / \mathrm{ml}$ (for PPD-type MaG and PPT-type $\mathrm{MaG}$ ) and $8 \mathrm{mg} / \mathrm{ml}$ (for Total-MaG) by preparing filtersterilized methanol stock of each fraction and mixing this with the appropriate volume of autoclaved, concentrated V8 agar before solidification of the agar. Methanol was used at a concentration of $1 \%$ of the total volume of the media containing ginsenosides as well as the control (Zhao et al. 2012). Three-millimeter-diameter plugs were taken from the edge of an actively growing colony of each isolate and transferred to the center of 6-cm-diameter plates of V8 agar containing either Total MaG, PPD-type MaG, or PPT-type $\mathrm{MaG}$ at the concentrations listed above. A plug of each isolate was transferred to ginsenoside-free V8 agar as a control. Two samples per treatment were used as replicates and the experiment was conducted three times. Inoculated plates were incubated in the dark at $25^{\circ} \mathrm{C}$ and growth rate was measured when the colony diameter on either control or ginsenoside-containing plates reached $80 \%$ of the plate size. The growth rate of each isolate was assessed using the following formula: $100-[(\mathrm{dc}-\mathrm{dt}) /(\mathrm{dc}-3 \mathrm{~mm}) \times 100](\mathrm{dc}=$ fungal colony diameter of control; $\mathrm{dt}=$ fungal colony diameter with ginsenoside treatment).

In vitro ginsenoside metabolism assay. Isolates of the I. radicicola species complex and Trichoderma spp. were grown on PDA media for 12 days at $25^{\circ} \mathrm{C}$ in the dark. Then, 3-mm plugs were taken from the actively growing edge of each isolate colony and transferred to 1.5 -ml centrifuge tubes containing $300 \mu \mathrm{l}$ of V8 broth supplemented with either Total MaG (8 mg/ml), PPD-type MaG (4 mg/ml), or PPT-type MaG (4 mg/ml), followed by incubation at $25^{\circ} \mathrm{C}$ in the dark with shaking $(150 \mathrm{rpm})$ for 20 days. Fractions containing V8 broth were inoculated with fungi-free plugs and incubated under the same conditions as the control. After incubation, ginsenosides were extracted by three times adding an equal volume of water saturated $n$-butanol. Then, butanol fractions were evaporated using a rotary evaporator and dried samples were redissolved in $1 \mathrm{ml}$ of HPLC-grade methanol, filtered through $0.2-\mu \mathrm{m}$ filters, and subjected to HPLC analysis for ginsenoside quantification. The experiment was conducted three different times. The ability of the isolates to metabolize ginsenosides in the three prepared fractions was evaluated by calculating the ginsenoside

TABLE 1. Ilyonectria radicicola species complex isolates used in this study, obtained from Korean ginseng roots

\begin{tabular}{|c|c|c|c|c|}
\hline Name & Isolate number & Collection date & Location & $\begin{array}{l}\text { GenBank accession } \\
\text { numbers of HIS H3 }\end{array}$ \\
\hline \multirow[t]{5}{*}{ I. robusta } & HB 1 & In this study & Yeoncheon, Korea & KP761762 \\
\hline & HB 2 & In this study & Yongin, Korea & KP761766 \\
\hline & HB 3 & In this study & Yeoncheon, Korea & KP761763 \\
\hline & HB 4 & In this study & Gochung, Korea & KP761764 \\
\hline & HB 5 & In this study & Gochung, Korea & KP761765 \\
\hline \multirow[t]{3}{*}{ I. leucospermi } & HB 6 (KACC 44655) & S. G. Lee 2004 & Yecheon, Gyeongbuk, Korea & KP761772 \\
\hline & HB 7 (KACC 44656) & S. G. Lee 2009 & Punggi, Yeongju, Gyeongbuk, Korea & KP761773 \\
\hline & HB 8 (KACC 44657) & S. G. Lee 2004 & Gamcheon, Yecheon, Gyeongbuk, Korea & KP761774 \\
\hline \multirow[t]{6}{*}{ I. mors-panacis } & HB 9 (KACC 44658) & S. G. Lee 2002 & Suwon, Gyeonggi, Korea & KP761767 \\
\hline & HB 10 (KACC 44659) & S. G. Lee 2000 & Suwon, Gyeonggi, Korea & KP761768 \\
\hline & HB 11 (KACC 44660) & S. G. Lee 1998 & Suwon, Gyeonggi, Korea & KP761769 \\
\hline & HB 12 (KACC 41077) & Y. S. Bae 2002 & Anseong, Gyeonggi, Korea & KP761775 \\
\hline & HB 13 (NBRC 31881) & Y. Miyazawa 1969 & Kitasaku-gun, Nagano Pref., Japan & KP761770 \\
\hline & HB 14 & In this study & Yeoncheon, South Korea & KP761771 \\
\hline
\end{tabular}


conversion efficiency $\left[\mathrm{GCE}=1-(\text { major ginsenosides })_{\text {treated }} /(\right.$ major ginsenosides) $\left.)_{\text {control }}\right)$ ].

Ginsenoside analysis. Ginsenosides were analyzed using an HPLC system (Agilent, Palo Alto, CA) as described by Kim et al. (2013) with some modifications; the device was equipped with an autosampler, a UV detector, and a C18 $(50 \times 4.6 \mathrm{~mm}$, Kinetex $2.6 \mathrm{u}$ C18 100A) column. Gradient elution was performed using solvent A ( $100 \%$ acetonitrile) and solvent B (100\% water) at $38^{\circ} \mathrm{C}$ using the following gradient program: 0 to $4 \mathrm{~min}, 19 \% \mathrm{~A}$ (isocratic); 4 to $9 \mathrm{~min}, 19$ to $25 \% \mathrm{~A} ; 9$ to $20 \mathrm{~min}, 25$ to $40 \% \mathrm{~A} ; 20$ to $25 \mathrm{~min}, 40$ to $56 \% ; 25$ to $28 \mathrm{~min}, 56$ to $70 \% \mathrm{~A} ; 28$ to $29 \mathrm{~min}, 70$ to $100 \% \mathrm{~A} ; 29$ to $35 \mathrm{~min}, 100 \%$ A (isocratic); 35 to $36 \mathrm{~min}, 100$ to $19 \% \mathrm{~A} ; 36$ to $42 \mathrm{~min}, 19 \%$ A (isocratic). The flow rate was kept at $1.2 \mathrm{ml} / \mathrm{min}$, the sample injection volume was $5 \mu \mathrm{l}$, and UV absorption was measured at $203 \mathrm{~nm}$. Quantitative analysis was performed using a one-point curve method using ginsenosides standards.

Statistical analyses. The pathogenicity, growth rate, and in vitro ginsenoside metabolism values of isolates of each identified species of Ilyonectria were averaged and are presented as the means of three different experimental replicates \pm standard errors (SE). All experimental data were analyzed via analysis of variance followed by protected least significant difference comparison of means $(P<$ 0.05) using XLSTAT software 2015.1.

\section{RESULTS}

Molecular identification and determination of $I$. morspanacis isolates. Fourteen isolates used in this study belonging to I. radicicola species complex were identified based on their HIS H3 sequence. Accordingly, six of those isolates were identified as the aggressive species, I. mors-panacis. Other isolates were resolved into two different species; five isolates were identified as I. robusta and three as I. leucospermi.

Ginseng root infection and ginsenoside profile. The identified isolates were soil-inoculated and their ability to infect ginseng roots of similar shape, size, and weight was investigated. Infected roots were harvested after 4 weeks, which was when symptoms (wilting and yellowing of the green part of germinated seedlings or inability of seedlings to germinate) were observed on seedlings infected with some isolates of the $I$. radicicola species complex compared with untreated control seedlings and seedlings infected with Trichoderma spp. No discoloration developed on the root part of untreated control or Trichoderma spp.-infected seedlings and they stayed rigid until harvesting time (Fig. 2A and $\mathrm{B})$, which confirmed that the softening and discoloration of treated roots was due to the inoculated Ilyonectria species. The results were normalized by calculating disease severity index (DSI). As expected, isolates of I. mors-panacis had significantly higher DSI values than isolates of I. robusta and I. leucospermi, as well as the untreated control and Trichoderma spp. (Table 2). Roots infected with I. mors-panacis showed root softening, discoloration, and inability to germinate (Fig. 2E). Isolates of other species (I. robusta and I. leucospermi) caused weak germination of seedlings and softening of less than half of the root length (Fig. 2C and D). The DSI values of the $I$. robusta and I. leucospermi isolates were significantly higher than those of the untreated control and Trichoderma spp. isolate; however, they were not higher than those of I. mors-panacis (Table 2). Ginsenoside content of the treated roots as well as of the untreated control was quantified to determine the effect of isolates' infection. Interestingly, Total MaG (including PPD and PPT types) content was significantly reduced in roots infected with $I$. mors-panacis isolates compared with those infected with other species, I. robusta and I. leucospermi, or Trichoderma spp. or the untreated control. In contrast, roots infected with I. robusta, I. leucospermi, and Trichoderma spp. isolates had a significantly higher concentration of Total MaG (including PPD and PPT types) than those infected with I. mors-panacis isolates and the untreated control (Fig. 3B). The dynamic changes in major ginsenosides can be seen in the HPLC profile for I. mors-panacis isolate HB 11 compared with those of the untreated control, I. robusta $\mathrm{HB} 4$, and I. leucospermi $\mathrm{HB} 8$ (Fig. 3A).

Preparation of ginsenoside fractions. Due to the reduction and increment of ginsenosides content after infection of the ginseng roots by I. mors-panacis and other fungi, respectively, the interaction between the major ginsenosides and all fungi was investigated in vitro. For this reason, pure fractions of total, PPDtype, and PPT-type MaGs were successfully purified from the saponin extract made from white ginseng. Total $\mathrm{MaG}$ fraction comprised the seven common ginsenosides: Rb1 1, Rb2 2, Rc 3, Rd 4, Rg1 11, Re 10, and Rf 12 (Fig. 4A). PPD-type MaG fraction comprised ginsenosides Rb1 1, Rb2 2, Rc 3, and Rd 4 (Fig. 4C), while the PPT-type MaG fraction comprised Rg1 11, Re 10, and Rf 12 (Fig. 4B).

Antifungal activity of ginsenosides. All Ilyonectria species tested and the Trichoderma spp. were sensitive to the Total MaG fraction. I. leucospermi had the lowest sensitivity, followed by I. robusta, with growth rate values over $80 \%$, while I. mors-panacis and Trichoderma spp. were more sensitive with growth rate values lower than $70 \%$. Growth rates of I. robusta and I. leucospermi were significantly higher on PPD-type MaG than on Total and PPT-type MaG. In contrast, the growth rate of I. mors-panacis on media containing the PPD and PPT-type MaG fractions was significantly
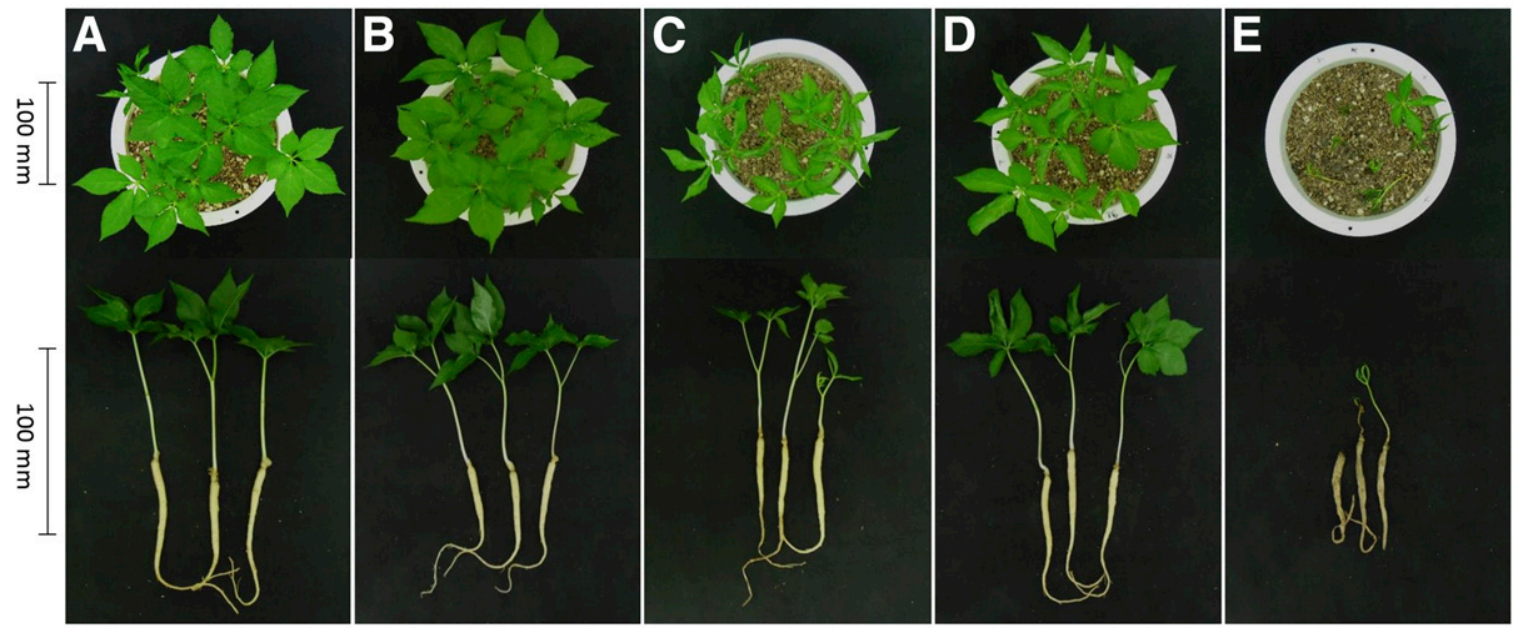

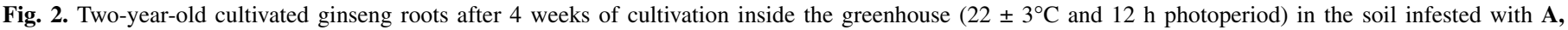
sterilized water (control), B, Trichoderma spp., C, Ilyonectria robusta HB 4, D, I. leucospermi HB 7, and E, I. mors-panacis HB 11. 
lower than growth of that on media supplemented with the Total $\mathrm{MaG}$ fraction, while the growth rate of Trichoderma spp. on the three fractions was not significantly different (Fig. 5). The results for the individual isolates are summarized in Supplementary Table S2.

In vitro metabolism of ginsenosides. The ability of the isolates to metabolize the three different ginsenosides fractions were tested in vitro to confirm that the dynamic changes in growth rate investigated before were due to the parental ginsenosides. Each isolate was inoculated directly into ginsenoside fraction-containing V8 broth and the remaining major ginsenosides (MaG) and accumulated minor ginsenosides $(\mathrm{MiG})$ were then compared with the untreated control using HPLC. In the HPLC profile of media supplemented with Total MaG and then inoculated with I. robusta and I. leucospermi isolates, the peaks of MaG, Rb1 1, Rb2 2, Rc 3, and $\operatorname{Rd} 4, \operatorname{Rg} 111$, and $\operatorname{Re} 10$, were partially reduced while the peaks of MiG, c-MC 7, c-Y 6, c-K 8, Rg2 14, and Rh1 13, were almost raised (Fig. 6B and $\mathrm{C}$ ). In the sample inoculated with I. morspanacis (Fig. 6D), the peaks were similar to those recorded for the untreated control (Fig. 6A). HPLC profile of media supplemented with Total MaG and inoculated with Trichoderma spp. was not different from that of the untreated control except for a decrease in the Rb1 1 peak and an increase in the Rd 4 peak; however, no accumulation of MiG was observed (Fig. 6E). The HPLC profile of PPT-type MaG was similar to that observed for the Total MaG fraction for each species isolate as well as Trichoderma spp. compared with the untreated control (Supplementary Fig. S2), but the HPLC profile of media supplemented with PPD-type MaG was different, especially in those inoculated with I. robusta and I. leucospermi isolates; the peaks of $\mathrm{MaG}$ disappeared and there was a large increase in the MiG peaks (Supplementary Fig. S3B and C). The HPLC profile of PPD-type MaG media inoculated with Trichoderma spp. was altered in the same way as the Total MaG fraction media, while stayed similar to that of untreated control in samples inoculated with I. mors-panacis isolates. Qualitatively, $\mathrm{MaG}$ in the total fraction were significantly reduced and MiG were significantly increased in media inoculated with $I$. robusta and I. leucospermi isolates, while no significant reduction was observed for media inoculated with I. mors-panacis isolates or the Trichoderma spp. isolate. Highest reduction was observed in I. robusta-inoculated media followed by media inoculated with I. leucospermi isolates, the Trichoderma spp. isolate, and the I. mors-panacis isolates. The last two samples did not show any significant reduction compared with untreated control samples (Fig. 7A). The significant differences in the PPT-type MaG fraction were similar to those observed for media supplemented with Total $\mathrm{MaG}$, but the reduction in $\mathrm{MaG}$ and accumulation of $\mathrm{MiG}$ in the I. leucospermi-inoculated samples were significantly higher than those observed for the I. robusta-inoculated samples (Fig. 7B). In PPD-type MaG media inoculated with all Ilyonectria species, there was a significant reduction in $\mathrm{MaG}$ and accumulation of $\mathrm{MiG}$ while media inoculated with Trichoderma spp. had similar quantities to the untreated control. Highest reduction in PPD-type MaG was observed in media inoculated with I. robusta isolates and the lowest reduction was observed in media inoculated with $I$. mors-panacis isolates (Fig. 7C). The results obtained from the individual isolates

TABLE 2. Mean values of ginsenoside conversion efficiency (GCE) determined from the in vitro metabolism assay of the ginsenosides fractions; Total MaG, PPTtype MaG, and PPD-type MaG for Ilyonectria robusta, I. leucospermi, and I. mors-panacis isolates compared with control and saprophytic fungus, Trichoderma spp., and disease severity index (DSI) for roots inoculated with the same isolates ${ }^{\mathrm{z}}$

\begin{tabular}{|c|c|c|c|c|}
\hline \multirow[b]{2}{*}{ Treatment } & \multicolumn{3}{|c|}{ GCE } & \multirow[b]{2}{*}{ DSI } \\
\hline & Total MaG & PPT MaG & PPD MaG & \\
\hline Control & - & - & - & $1.61 \pm 0.068 \mathrm{c}$ \\
\hline Trichoderma spp. & $0.05 \pm 0.0117 \mathrm{c}$ & $0.05 \pm 0.0034 \mathrm{c}$ & $0.05 \pm 0.003 \mathrm{~d}$ & $1.50 \pm 0.117 \mathrm{c}$ \\
\hline I. robusta & $0.31 \pm 0.0217 \mathrm{a}$ & $0.08 \pm 0.0323 b$ & $0.85 \pm 0.0199 a$ & $1.95 \pm 0.013 b$ \\
\hline I. leucospermi & $0.25 \pm 0.0157 \mathrm{~b}$ & $0.21 \pm 0.0094 a$ & $0.75 \pm 0.0003 b$ & $1.85 \pm 0.098 b$ \\
\hline I. mors-panacis & $0.03 \pm 0.0058 \mathrm{c}$ & $-0.13 \pm 0.0155 \mathrm{~d}$ & $00.14 \pm 0.0095 \mathrm{c}$ & $4.99 \pm 0.064 \mathrm{a}$ \\
\hline
\end{tabular}

z Abbreviations: Total MaG, total major ginsenosides; PPT-type MaG, PPT-type major ginsenosides; and PPD-type MaG, PPD-type major ginsenosides. Values are expressed as mean \pm SE. Data followed by the same letter are not significantly different $(P<0.05$, least significant difference test, $n=3)$.
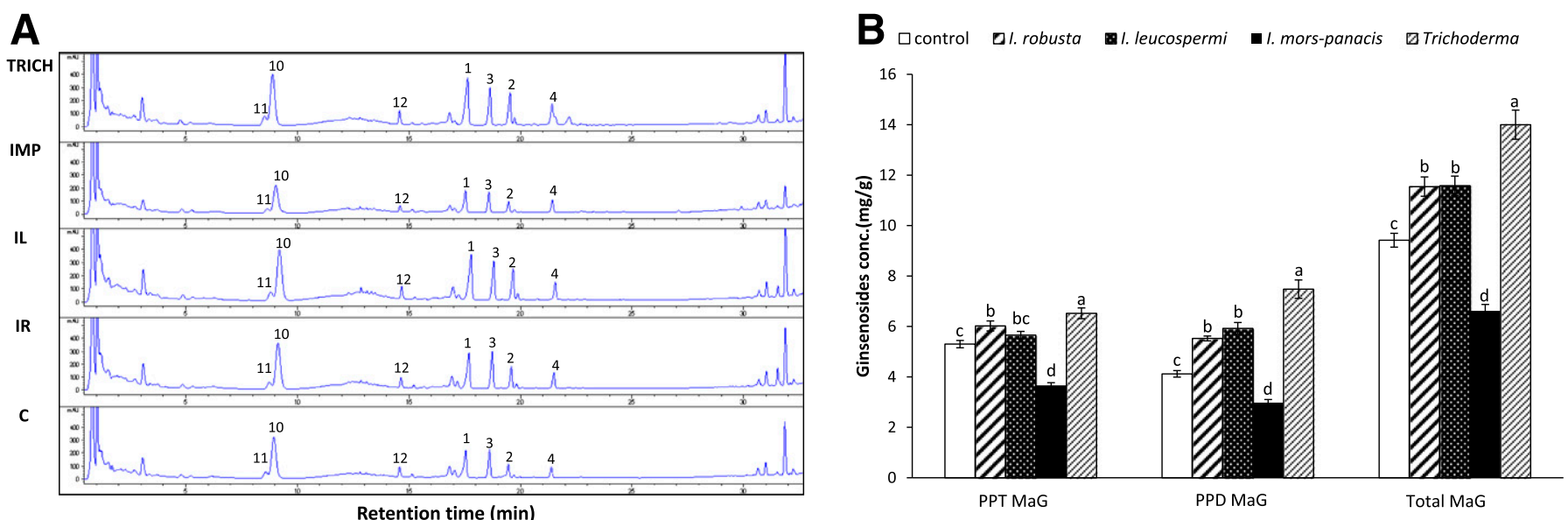

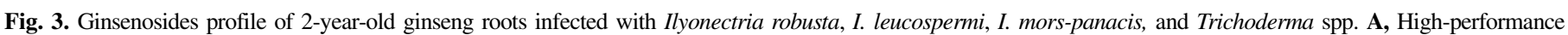

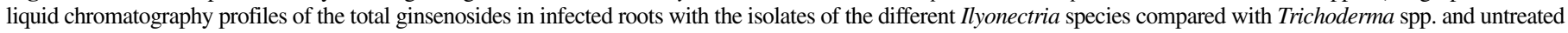

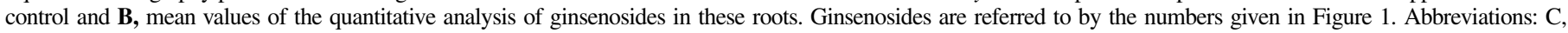

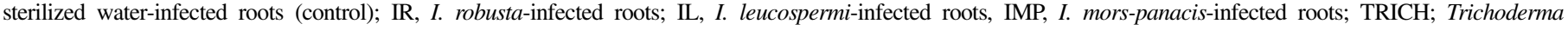

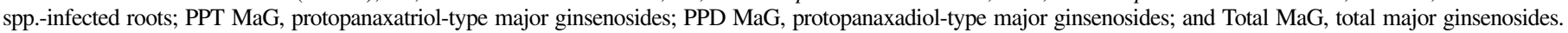
Values are expressed as mean \pm SE. Data columns with the same letter are not significantly different $(P<0.05$, least significant difference test, $n=3)$ 
are summarized in Supplementary Table S3. Furthermore, the ability of each isolate to metabolize ginsenosides in the three fractions was normalized by calculating GCE (Ivanov and Bernards 2012). Accordingly, in the PPD-type MaG fraction, I. robusta isolates had the highest significant GCE value, followed by I. leucospermi, I. mors-panacis, and Trichoderma spp. For the PPT-type and total fractions, GCE decreased drastically. The highest significant value for the PPT-type fraction was recorded for I. leucospermi, followed by I. robusta and Trichoderma spp., while the lowest value was recorded for I. mors-panacis. For media supplemented with the total fraction, the highest significant value was recorded for I. robusta, followed by I. leucospermi, while the lowest value was recorded for I. mors-panacis, which did not show any significant difference with that of Trichoderma spp. (Table 2). The GCE values of the individual isolates for each fraction are summarized in Supplementary Table S4.

\section{DISCUSSION}

In the current study, we identified 14 isolates from Korean ginseng belonging to the I. radicicola species complex, according to the sequence of HIS H3 (Cabral et al. 2011). Out of them, six were identified as the aggressive species, I. mors-panacis, while the remaining were resolved into two species, I. robusta and I. morspanacis. This is consistent with the study of Cabral et al. 2011; those authors reclassified 22 isolates of the I. radicicola species complex isolated from American ginseng into four species. Out of them, 16 isolates were characterized as I. mors-panacis, while the remaining were resolved into three different species including $I$. robusta. The first report of I. robusta on Korean ginseng in China was recently published ( $\mathrm{Lu}$ et al. 2015). I. leucospermi has previously been detected in vegetative cuttings from Protea and Leucospermum
(Lombard et al. 2013), but has not previously been reported for Panax species. Our study is therefore the first report of I. leucospermi on Panax ginseng.

The diversity of pathogenicity in the $I$. radicicola species complex is of great interest to ginseng growers. Pathogenicity tests of diverse isolates of $C$. destructans from ginseng and other hosts demonstrated the presence of a group of isolates that were highly aggressive on ginseng only, and all of these isolates were isolated only from ginseng (Rahman and Punja 2005; Seifert et al. 2003). These isolates were named $C$. destructans f. sp. panacis (Seifert

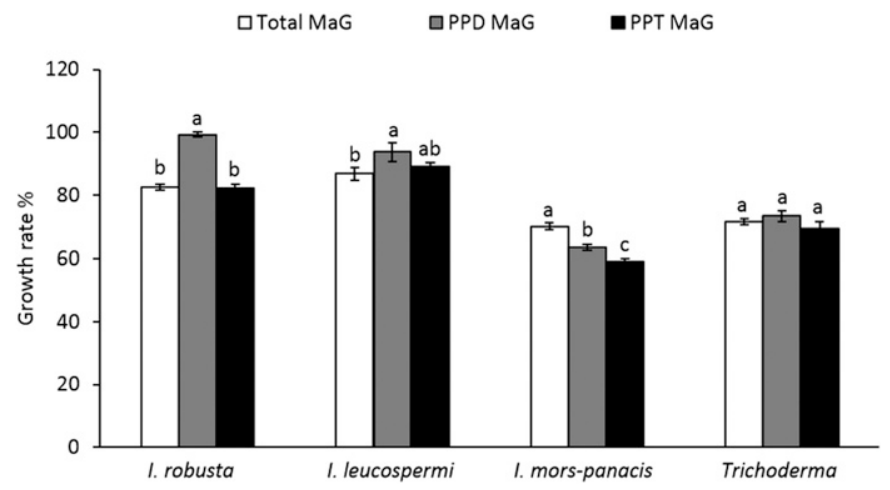

Fig. 5. Mean values of the growth rate of Ilyonectria robusta, I. leucospermi, and I. mors-panacis isolates compared with Trichoderma spp., after growing on V8 agar media amended with different fractions of ginsenosides. Abbreviations: Total $\mathrm{MaG}$, total major ginsenosides; PPD MaG, protopanaxadioltype major ginsenosides; PPT MaG, protopanaxatriol-type major ginsenosides. Values are expressed as mean \pm SE. Same letters indicate no significances $(P<$ 0.05 , least significant difference test, $n=3$ ).

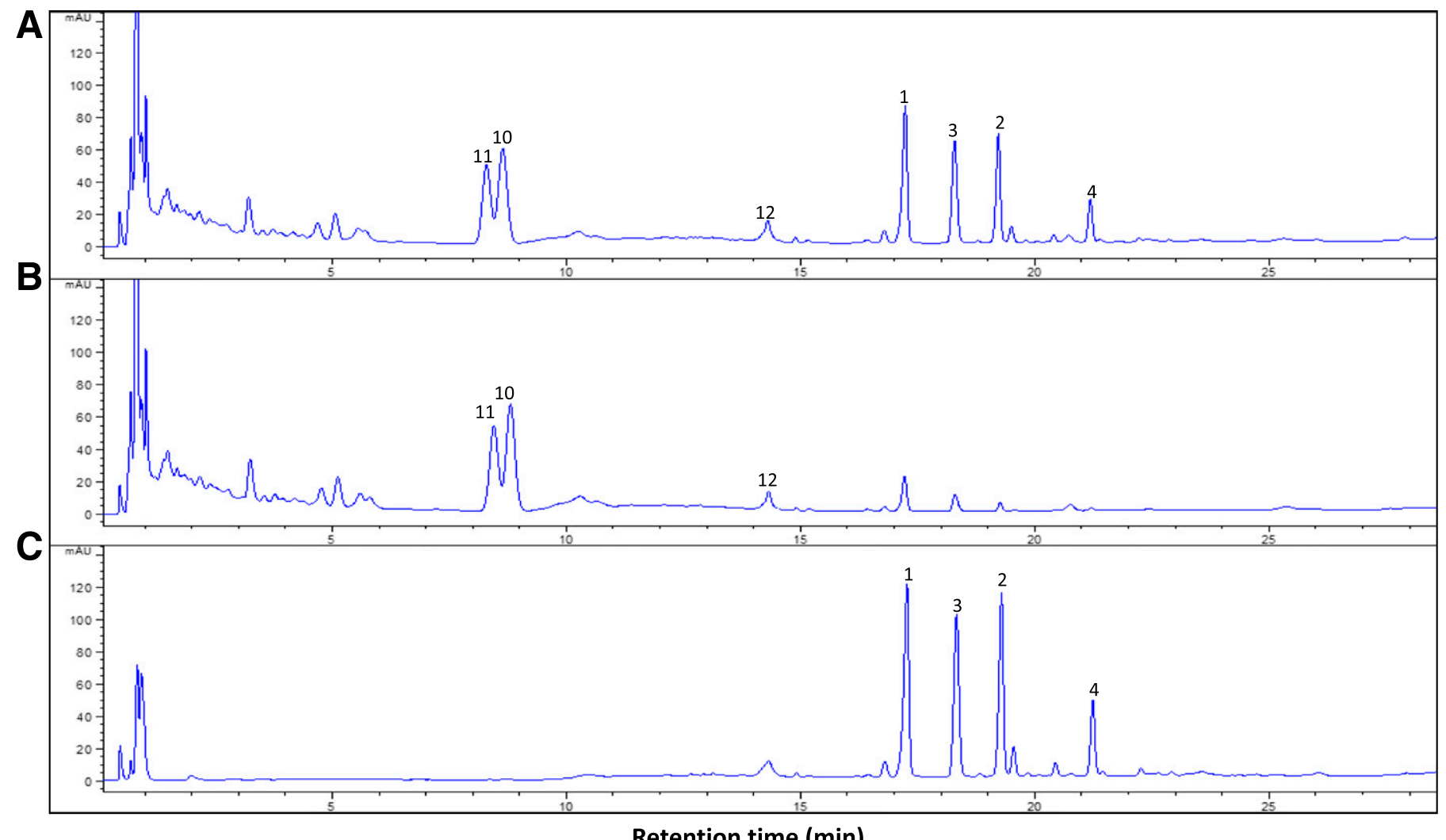

Retention time (min)

Fig. 4. High-performance liquid chromatography analysis representing A, Total MaG, B, PPT-type MaG, and C, PPD-type MaG (2, 1 , and 1.9 mg/ml, respectively), that were prepared from white ginseng saponin extract for in vitro interaction between saponins and fungi. Ginsenosides are referred to by the numbers given in Figure 1. Abbreviations: Total MaG, total major ginsenosides; PPT-type MaG, protopanaxatriol-type major ginsenosides; and PPD-type MaG, protopanaxadiol-type major ginsenosides. 


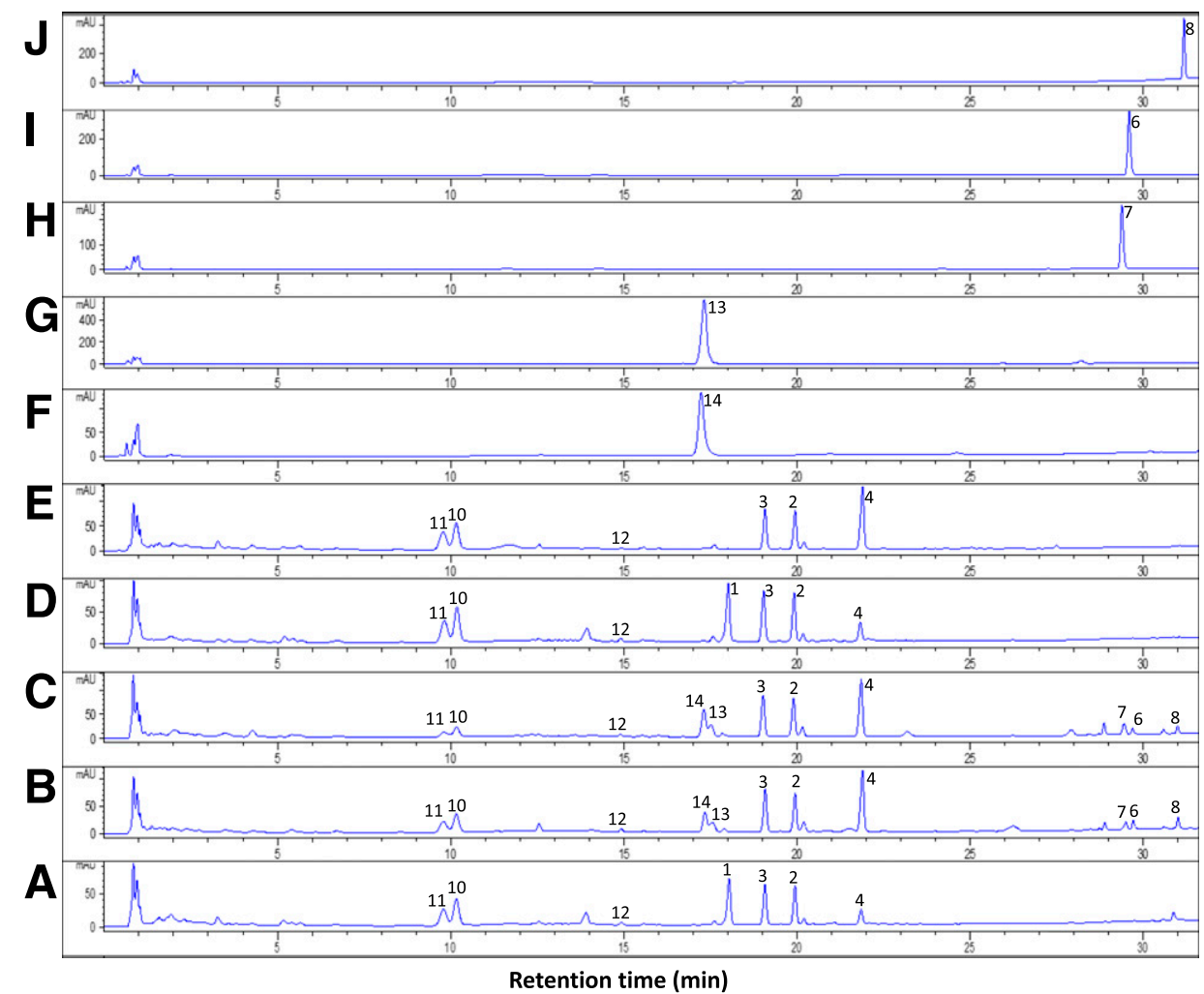

Fig. 6. Representative high-performance liquid chromatography profiles of total major ginsenosides incubated for 20 days at $25^{\circ} \mathrm{C}$ after inoculation with $\mathbf{A}$, fungusfree agar (control), B, Ilyonectria robusta $\mathrm{HB}$ 4, C, I. leucospermi HB 7, D, I. mors-panacis HB 11, and E, Trichoderma spp. F to J, Represent the standards of the minor ginsenosides Rg2 14, Rh1 13, c-MC 7, c-Y 6, and c-K 8, respectively. Ginsenosides are referred to by the numbers given in Figure 1. Abbreviations: c-MC, compound MC; c-Y, compound $\mathrm{Y}$; and $\mathrm{c}-\mathrm{K}$, compound $\mathrm{K}$.

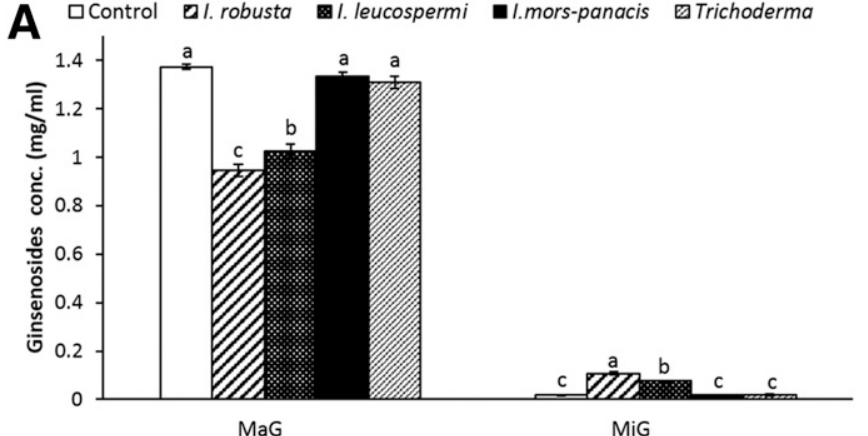

MaG

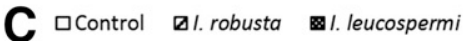

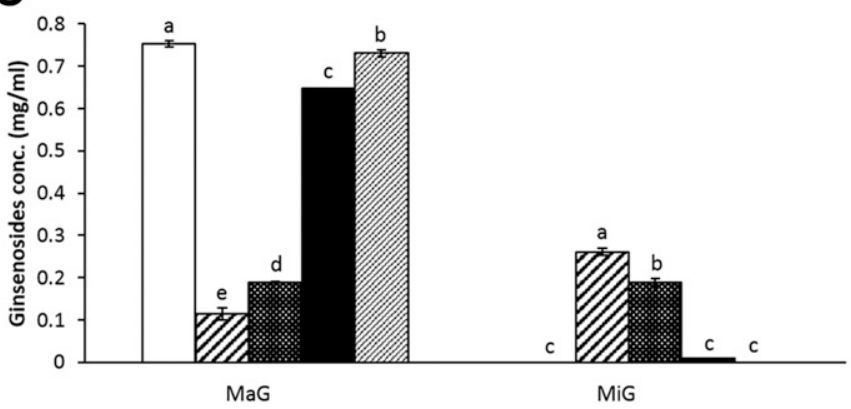

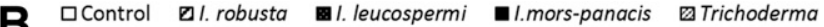

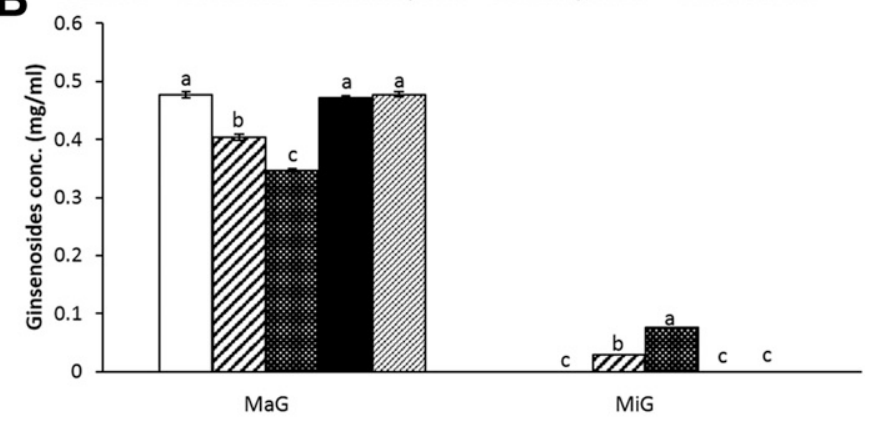

Fig. 7. Quantitative analysis of A, total, B, PPT-type, and C, PPD-type ginsenosides fractions by high-performance liquid chromatography, showing major ginsenosides depletion and minor ginsenosides accumulation in V8 broth amended with total major ginsenosides ( $8 \mathrm{mg} / \mathrm{ml})$, PPT-type major ginsenosides (4 mg/ml), and PPD-type major ginsenosides $(4 \mathrm{mg} / \mathrm{ml}$ ) inoculated with fungus-free agar (control), Ilyonectria robusta, I. leucospermi, I. mors-panacis, and Trichoderma spp. for 20 days. Abbreviations: PPT-type, protopanaxatriol-type; PPD-type, protopanaxadiol-type; MaG, major ginsenosides; and MiG, minor ginsenosides. Values are expressed as mean $\pm \mathrm{SE}$. Data columns with the same letter are not significantly different $(P<0.05$, least significant difference test, $n=3)$. 
et al. 2003). Multigene analysis revealed that these isolates were genetically distinct, and they were therefore defined as a species, I. mors-panacis (Cabral et al. 2011). We initially evaluated the pathogenicity of all isolates by the root assay method. Roots infected with I. mors-panacis isolates, however, did not show any disease symptoms and only $I$. robusta isolates induced root discoloration and rotting. It is possible that isolates of I. morspanacis can survive only under soil conditions, while I. robusta causes root discoloration by growing saprophytically on the roots (data not shown). This is supported by a previous study that reported contrasting data when using a soil system for assessing isolates pathogenicity (Seifert et al. 2003). We therefore evaluated pathogenicity in the present study using soil conditions. The results of pathogenicity test are in agreement with previous reports; all highly aggressive isolates (DSI values $>4$ ) belonged to I. morspanacis. According to Koch's postulates, the I. mors-panacis isolates rather than other fungi were responsible for the observed root rotting. The other identified species, I. robusta and I. crassa, which are considered weakly aggressive isolates of $C$. destructans in ginseng roots (Seifert et al. 2003), were genetically similar to those infecting other hosts (Cabral et al. 2011). Also, in this study, strains $\mathrm{HB} 6, \mathrm{HB} 7$, and $\mathrm{HB} 8$, which were recorded as weakly aggressive isolates, were genetically similar to those infecting Protea and Leucospermum (Lombard et al. 2013). These findings suggest that $I$. mors-panacis has a specific interaction with its sole host, ginseng, leading to severe infection. The other species may not be aggressive because ginseng is not their original host.

Saponins are antifungal compounds that are thought to be produced by plants to prohibit plant infection by soilborne fungi. Even though they are constitutively produced, levels of these saponins increase in response to fungal attack (Augustin et al. 2011; Pegg and Woodward 1986). Ginsenoside concentrations were shown to increase in adventitious hairy roots of Panax ginseng after treatment with fungal elicitors (Liu et al. 2004). In the present study, a significant increase in Total MaG was observed in ginseng roots infected with the weakly aggressive species, I. robusta and I. leucospermi, as well as a Trichoderma spp. isolate. This suggests that Total $\mathrm{MaG}$ production could be a defensive response to fungal attack and that Total MaG may function as antifungal agents to reduce fungal invasion. Levels of both PPT and PPD-type MaG were significantly increased. Therefore, both types may be involved in plant defense. In contrast, a significant reduction in Total $\mathrm{MaG}$ (both PPT and PPD types) was observed in roots infected with the aggressive I. mors-panacis isolates. A reduction in the tomato saponin, $\alpha$-tomatine, was observed after artificial inoculation of tomato with the blight spot-causing pathogen, Cladosporium fulvum. This pathogen was found to resist the antifungal activity of $\alpha$-tomatine through production of $\alpha$-tomatine-hydrolyzing $\beta$-glucosidase, which resulted in accumulation of a less fungitoxic compound (Ökmen et al. 2013). Initially, we hypothesized that I. mors-panacis isolates may use a similar mechanism to reduce the toxicity of ginsenosides, but the growth of the aggressive species, I. mors-panacis, was significantly reduced in media supplemented with any of the three different ginsenoside fractions, particularly PPT-type MaG. In addition, the ability of I. mors-panacis isolates to metabolize ginsenosides in the three fractions, as indicated by GCE values, was $<0.14$. This suggests that this aggressive species is sensitive to naturally-occurring ginsenosides, particularly PPT-type, and that production of ginsenoside-hydrolyzing $\beta$ glucosidases is not the mechanism by which ginsenoside content was reduced. Ustilago maydis, the hemibiotrophic pathogen that causes smut disease in maize, can establish a successful infection by suppressing the production of reactive oxygen species (ROS) such as $\mathrm{H}_{2} \mathrm{O}_{2}$. The supposed mechanism is the following: the pathogen produces an effector called Pep1 in the plant apoplast, which binds to and inhibits maize peroxidase (POX12), leading to suppression of ROS production and establishment of efficient pathogen penetration (Hemetsberger et al. 2012). Furthermore, U. maydis suppresses the production of plant salicylic acid (SA), which signals the plant immune system: the pathogen produces Cmu1, a functional chorismate mutase enzyme that transform chorismate, the key intermediate in SA biosynthesis (Wildermuth et al. 2001), to prephenate (Djamei et al. 2011), thereby suppressing SA accumulation. The key enzymes controlling the ginsenoside pathway are squalene synthase, squalene epoxidase, dammarenediol-II synthase, cytochrome P450, and several glucosyltransferases (Wang et al. 2012). Also, ginsenosides production is signaled by jasmonic acid (JA) (Yu et al. 2002) which is elicited by SA (Rahimi et al. 2014). Because I. mors-panacis is host specific, we hypothesized that I. mors-panacis may similarly suppress ginsenoside production by either inhibiting the function of one or more of those key enzymes or suppressing the accumulation of SA.

The growth of I. robusta and I. leucospermi isolates on media containing Total and PPT-type MaG was significantly reduced, although these isolates were less sensitive to these fractions than the I. mors-panacis isolates. Growth of I. robusta and I. leucospermi isolates was higher on media supplemented with PPD-type MaG than media supplemented with Total or PPT-type MaG. GCE values of I. robusta and I. leucospermi isolates grown on media containing the PPD-type $\mathrm{MaG}$ fraction were high, but their GCE values on media supplemented with PPT-type MaG or Total MaG fractions were lower. Growth induction of a C. destructans isolate by PPD-type exposure and reduction by PPT-type exposure (Zhao et al. 2012) is consistent with the results of $I$. robusta and I. leucospermi which may indicate that this isolate is not I. mors-panacis. Based on these observations, PPD-type MaG appears to be less fungitoxic to these species than PPT-type MaG; the latter may work separately or synergistically with PPD-type MaG to reduce fungal attack. Solanum species produce two types of saponins, one of which is more biologically effective than the other. One of these saponins is thought to be produced to suppress pathogen attack, while the other saponin is produced by modifying the position of a sugar residue on the first saponin after the pathogen has evolved to overcome the antifungal activities of the former saponin (Friedman and McDonald 1999; Roddick et al. 2001). PPD-type ginsenosides may be the original saponins produced by ginseng. Pathogen adaptation over time may have allowed the pathogens to become resistant to PPD-type ginsenosides, possibly by hydrolyzing the sugar residues, resulting in production of more potent PPT-type antifungal ginsenosides. Based on that, it is assumed that the sensitivity of $I$. robusta and I. leucospermi to PPT-type MaG might be the reason for their low pathogenicity.

In conclusion, our study explored the antifungal activity of the ginsenosides, in particular PPT-type, which may intervene in the plant defense mechanism against Ilyonectria infecting Korean ginseng, in particular the weakly aggressive species. In addition, the pathogenicity of the aggressive species, I. mors-panacis, may depend on the reduction of ginseng saponins. However, as the pathogenicity does not correlate with the ability to metabolize the major ginsenosides, the reduction of ginsenosides may occur via a mechanism other than detoxification. Further experimental work is needed to explore this mechanism.

\section{ACKNOWLEDGMENTS}

This study was supported by a grant from the Next-Generation BioGreen 21 Program (SSAC, grant PJ0120342016), Rural Development Administration, Republic of Korea.

\section{LITERATURE CITED}

Armah, C. N., Mackie, A. R., Roy, C., Price, K., Osbourn, A. E., Bawyer, P., and Ladha, S. 1999. The membrane permeabilising effect of avenacin A-1 involves the reorganisation of bilayer cholesterol. Biophys. J. 76:281-290. 
Augustin, J. M., Kuzina, V., Andersen, S. B., and Bak, S. 2011. Molecular activities, biosynthesis and evolution of triterpenoid saponins. Phytochemistry 72:435-457.

Booth, C. 1966. The Genus Cylindrocarpon. Page 104. Mycol. CMI, Kew, Surrey, England.

Cabral, A., Groenewald, J. Z., Rego, C., Oliveira, H., and Crous, P. W. 2011. Cylindrocarpon root rot: Multi-gene analysis reveals novel species within the Ilyonectria radicicola species complex. Mycol. Progress 11:665-688.

Chaverri, P., Salgado, C., Hirooka, Y., Rossman, A. Y., and Samuels, G. J. 2011. Delimitation of Neonectria and Cylindrocarpon (Nectriaceae, Hypocreales, Ascomycota) and related genera with Cylindrocarpon like anamorphs. Stud. Mycol. 68:57-78.

Crombie, W. M. L., Crombie, L., Green, J. B., and Lucas, J. A. 1986. Pathogenicity of the take all fungus to oats: Its relationship to the concentration and detoxification of the four avenacins. Phytochemistry 25:2075-2083.

Crous, P. W., Groenewald, J. Z., Risede, J. M., and Hywel-Jones, N. L. 2004. Calonectria species and their Cylindrocladium anamorphs: Species with sphaeropedunculate vesicles. Stud. Mycol. 50:415-429.

Djamei, A., Schipper, K., Rabe, F., Ghosh, A., Vincon, V., and Kahnt, J. 2011. Metabolic priming by a secreted fungal effector. Nature 478:395-398.

Fewell, A. M., and Roddick, J. G. 1993. Interactive antifungal activity of the glycoalkaloid solanine and chaconine. Phytochemistry 33:323-328.

Ford, J. E., McChance, D. J., and Drysdale, R. B. 1977. The detoxification of atomatine by Fusarium oxysporum f. sp. lycopersici. Phytochemistry 16: 545-546.

Friedman, M., and McDonald, G. M. 1999. Steroidal glycoalkaloids. Pages 311-343 in: Naturally Occurring Glycosides. R. Ikan, ed. Wiley \& Sons, New York.

Hemetsberger, C., Herrberger, C., Zechmann, B., Hillmer, M., and Doehlemann, G. 2012. The Ustilago maydis effector Pep1 suppresses plant immunity by inhibition of host peroxidase activity. PLoS Pathog. 8: e1002684.

Hildebrand, A. A. 1935. Root rot of ginseng in Ontario caused by members of the genus Ramularia. Can. J. Res. 12:82-114.

Ivanov, D. A., and Bernards, M. A. 2012. Ginsenosidases and the pathogenicity of Pythium irregulare. Phytochemistry 78:44-53.

Kernaghan, G., Reeleder, R. D., and Hoke, S. M. T. 2007. Quantification of Cylindrocarpon destructans $\mathrm{f}$. sp. panacis in soils by real-time PCR. Plant Pathol. 56:508-516.

Keukens, E. A. J., de Vrije, T., van den Boom, C., de Waard, P., Plasman, H. H., Thiel, F., Chupin, V., Jongen, W. M. F., and de Kruijff, B. 1995. Molecular basis of glycoalkaloid induced membrane disruption. Biochim. Biophys. Acta 1240:216-228.

Kim, Y. J., Jeon, J. N., Jang, M. G., Oh, J. Y., Kwon, W. S., Jung, S. K., and Yang, D. C. 2013. Ginsenoside profiles and related gene expression during foliation in Panax ginseng Meyer. J. Ginseng Res. 38:66-72.

Lairini, K., and Ruiz-Rubio, M. 1998. Detoxification of a-tomatine by Fusarium solani. Mycol. Res. 102:1375-1380.

Leung, K. W., and Wong, A. S. 2010. Pharmacology of ginsenosides: A literature review. Chin. Med. 5:20.

Liu, J., Ding, J. Y., Zhou, Q. Y., He, L., and Wang, Z. T. 2004. Studies on influence of fungal elicitor on hairy root of Panax ginseng biosynthesis ginseng saponin and biomass. Zhongguo Zhongyao Zazhi 29:302-305.

Lombard, L., Bezuidenhout, C. M., and Crous, P. W. 2013. Ilyonectria black foot rot associated with Proteaceae. Australas. Plant Pathol. 42:337-349.

Lu, X. H., Jiao, X. L., Chen, A. J., Luo, Y., and Gao, W. W. 2015. First report of Ilyonectria robusta causing rusty root of Asian ginseng in China. Plant Dis. 99:156.

Lyr, H., and Kluge, E. 1968. Zusammenhange zwischen Pathogenitat, Enzymund Toxinproduktion bei Cylindrocarpon radicicola. J. Phytopathol. 62: 220-231.

Morrissey, J. P., and Osbourn, A. E. 1999. Fungal resistance to plant antibiotics as a mechanism of pathogenesis. Microbiol. Mol. Biol. Rev. 63:708-724.

Nicol, R. W., Traquair, J. A., and Bernards, M. A. 2002. Ginsenosides as host resistance factors in American ginseng (Panax quinquefolius). Can. J. Bot. 80:557-562

Nicol, R. W., Yousef, L., Traquair, J. A., and Bernards, M. A. 2003. Ginsenosides stimulate the growth of soilborne pathogens of American ginseng. Phytochemistry 64:257-264.
Nirenberg, H. I. 1976. Untersuchungen u"ber die morphologische und biologische Differenzierung in der Fusarium-Section Liseola. Berlin-Dahlem: Mitt Biol Bundesanst für Land-und Forstwirt 169:1-117.

Ökmen, B., Etalo, D. W., Joosten, M. H., Bouwmeester, H. J., de Vos, R. C., Collemare, J., and de Wit, P. J. 2013. Detoxification of $\alpha$-tomatine by Cladosporium fulvum is required for full virulence on tomato. New Phytol. 198:1203-1214.

Osbourn, A. 1996. Saponins and plant defense-A soap story. Trends Plant Sci. $1: 4-9$.

Osbourn, A., Bowyer, P., Lunness, P., Clarke, B., and Daniels, M. 1995. Fungal pathogens of oat roots and tomato leaves employ closely related enzymes to detoxify different host plant saponins. Mol. Plant-Microbe Interact. 8: 971-978.

Papadopoulou, K., Melton, R. E., Leggett, M., Daniels, M. J., and Osbourn, A. E. 1999. Compromised disease resistance in saponin-deficient plants. Proc. Natl. Acad. Sci. USA 96:12923-12928.

Pegg, G. F., and Woodward, S. 1986. Synthesis and metabolism of a-tomatine in tomato Lycopersicon esculentum isolines in relation to resistance to Verticillium albo-atrum. Physiol. Mol. Plant Pathol. 28:187-201.

Rahimi, S., Devi, B. S. R., Khorolragchaa, A., Kim, Y. J., Kim, J. H., Jung, S. K., and Yang, D. C. 2014. Effect of salicylic acid and yeast extract on the accumulation of jasmonic acid and sesquiterpenoids in Panax ginseng adventitious roots. Russ. J. Plant Physiol. 61:811-817.

Rahman, M., and Punja, Z. K. 2005. Factors influencing development of root rot on ginseng caused by Cylindrocarpon destructans. Phytopathology 95 : 1381-1390.

Roddick, J. G., Weissenberg, M., and Leonard, A. L. 2001. Membrane disruption and enzyme inhibition by naturally-occurring and modified chacotriose-containing Solanum steroidal glycoalkaloids. Phytochemistry 56:603-610.

Rossman, A. Y., Samuels, G. J., Rogerson, C. T., and Lowen, R. 1999. Genera of Bionectriaceae, Hypocreaceae and Nectriaceae (Hypocreales, Ascomycetes). Stud. Mycol. J. 42:1-248.

Seifert, K. A., McMullen, C. R., Yee, D., Reeleder, R. D., and Dobinson, K. F. 2003. Molecular differentiation and detection of ginseng-adapted isolates of the root rot fungus Cylindrocarpon destructans. Phytopathology 93: 1533-1542.

Shehzad, O., Ha, I. J., Park, Y., Ha, Y. W., and Kim, Y. S. 2011. Development of a rapid and convenient method to separate eight ginsenosides from Panax ginseng by high-speed counter-current chromatography coupled with evaporative light scattering detection. J. Sep. Sci. 34:1116-1122.

Siraj, F. M., Natarajan, S., Huq, M. A., Kim, Y. J., and Yang, D. C. 2015. Structural investigation of ginsenoside $\mathrm{Rf}$ with PPARg major transcriptional factor of adipogenesis and its impact on adipocyte. J. Ginseng Res. 39: 141-147.

Steel, C. C., and Drysdale, R. B. 1988. Electrolyte leakage from plant and fungal tissues and disruption of liposome membranes by $\alpha$-tomatine. Phytochemistry 27:1025-1030.

Sutherland, J. R., Shrimpton, G. M., and Surrock, R. N. 1989. Diseases and insects in British Columbia forest seedling nurseries. Forestry Canada and B.C. Ministry of Forests, Victoria. FRDA Report No. 065.

Unestam, T., Beyer-Ericson, L., and Strand, M. 1989. Involvement of Cylindrocarpon destructans in root death of Pinus sylvestris seedlings: Pathogenic behavior and predisposing factors. Scand. J. For. Res. 4:521-535.

Wang, J., Gao, W. Y., Zhang, J., Zuo, B. M., Zhang, L. M., and Huang, L. Q. 2012. Advances in study of ginsenoside biosynthesis pathway in Panax ginseng CA Meyer. Acta Physiol. Plant. 34:397-403.

Wildermuth, M. C., Dewdney, J., Wu, G., and Ausubel, F. M. 2001. Isochorismate synthase is required to synthesize salicylic acid for plant defense. Nature 414:562-565.

Yousef, L. F., and Bernards, M. A. 2006. In vitro metabolism of ginsenosides by the ginseng root pathogen Pythium irregulare. Phytochemistry 67: 1740-1749.

Yu, K. W., Gao, W., Hahn, E. J., and Paek, K. Y. 2002. Jasmonic acid improving ginsenoside accumulation in adventitious root culture of Panax ginseng C.A. Meyer. Biochem. Eng. J. 11:211-215.

Zhao, X., Gao, J., Song, C., Fang, Q., Wang, N., Zhao, T., Liu, D., and Zhou, Y. 2012. Fungal sensitivity to and enzymatic deglycosylation of ginsenosides. Phytochemistry 78:65-71. 